\title{
Analysing communication requirements for crowd sourced backend generation of HD Maps used in automated driving
}

\author{
Florian Jomrich*‡, Josef Schmid", Steffen Knapp*, Alfred Höß॥, Ralf Steinmetz ${ }^{\ddagger}$, Björn Schuller ${ }^{\S}$ \\ ${ }^{*}$ Opel Automobile GmbH, 65423 Rüsselsheim, Hessen, Germany \\ "East Bavarian Technical University Amberg-Weiden, 92224 Amberg, Bavaria, Germany \\ $\S$ ZD.B Chair of Embedded Intelligence for Health Care and Wellbeing, University of Augsburg, Germany \\ ${ }^{\ddagger}$ KOM Multimedia Communications Lab, Technical University of Darmstadt, 64283 Darmstadt, Hessen, Germany \\ Email: $\{\text { surname.name }\}^{*} @$ opel.com, ${ }^{\ddagger} @$ kom.tu-darmstadt.de, $\|\{$ j.schmid,a.hoess $\} @$ oth-aw.de,${ }^{\S}$ schuller@iee.org
}

\begin{abstract}
Highly automated vehicles rely on high-definition maps to ensure both safety and comfort of their passengers while driving. The maps provide a centimetre-accurate representation of the surrounding infrastructure to the car and ease tasks like localisation and object recognition by providing comparable reference material. Maintaining the maps with updates for the current traffic situation (e.g.traffic jams or construction work) is a challenging task. In the German government-funded research project Cooperative Highly Automated Driving (Ko-HAF) it is investigated to what extend vehicle sensors can be used to update these kinds of maps. In this work we present our obtained results and investigate the requirements for the cellular network infrastructure required for highly automated driving. To the best of our knowledge this work is one of the first that provides this correlation between data requirements and network infrastructure capabilities.
\end{abstract}

Index Terms-hd-map, updates, highly automated driving, cellular communication,

\section{INTRODUCTION}

Highly automated vehicles are going to change the future of our personal mobility. The vehicles will drive autonomously to any destination, freeing the human from this often tedious task, e.g. during traffic jams, to leave time to spend for other things. This includes recreational tasks like using the infotainment system of the car to stream videos and music or to browse the internet, as well as more productive tasks, like writing e-mails or attending video conferences, making the vehicle a mobile office. To be able to drive autonomously the vehicles have to rely on a multitude of sensors, like cameras, radar or lidar sensors [1] to observe their surrounding traffic situation.

The redundancy of sensors increases the vehicles safety while driving highly automated. Still, there are certain situations in which the car's sensor equipment is not enough to provide the passengers with a safe and comfortable driving experience. These are, for example, situations in which the sensor range is limited due to bad weather conditions (e.g. snow on traffic signs as well as rainy or foggy weather making it difficult for the camera to recognize objects). Furthermore, the general road network infrastructure can be problematic as well, tight turns and road hazards in concealed areas (e.g. a traffic jam behind a corner) cannot be compensated even with more enhanced on-board sensor equipment. To overcome these situations and to ensure safety and comfort of the passengers while driving, the car has to rely on a further "virtual" sensor, the so-called High-Defintion Map (HD Map). Compared to standard navigational map material, this map is much more accurate, down to the centimetre level. This way the map facilitates the vehicle's own driving and localisation task by providing accurate reference material. To achieve this high accuracy special vehicles are used to initially create the HD Map. Those cars can rely on a very precise sensor equipment, like Differental GPS (D-GPS), a multitude of cameras as well as highly accurate laser scanners, to collect the present obstacles and the traffic rules, which apply to the surrounding environment. However, the road network is not a static environment, but constantly affected by change, for example introduced through traffic jams, accidents, construction works or the current status of adaptive traffic signs. Thus, the map furthermore has to be continously updated to provide the car with up-to-date traffic information. This information, however, cannot be collected by the vehicles used to initially create the map. Their number is just to small and the required updates are to frequent. A very promising approach to still safely update the map is to rely on the self-driving vehicle's own sensor equipment to collect sensor readings from the current traffic situation and to provide these to a data processing back end via cellular communication. Through this crowdsourcing approach the back end server shall be able to process the data in a manner that satisfies both time and accuracy requirements of the highly automated vehicles. This concept is currently investigated by different research projects [2], [3], vehicle manufacturers [1], [4], map data providers [5], [6], [7] and many more (e.g see the group of SENSORIS members ${ }^{1}$ ). One of them is the German government-funded research project "Ko-HAF" (Cooperative Highly Automated Driving) ${ }^{2}$. The participants in Ko-HAF include several German OEMs and

\footnotetext{
${ }^{1}$ http://sensor-is.org/members/

${ }^{2}$ www.ko-haf.de
} 
Tier 1 suppliers, as well as affiliated researchers from different universities. The core of the project is a fleet of about a dozen cars that are able to collect and transmit sensor data to the Ko-HAF back end server to ensure and enhance their own highly automated driving capabilities. This paper illustrates the achieved results of Ko-HAF to shed light on the question of the actual requirements of the highly automated vehicles regarding data consumption and network traffic load. Furthermore, it also provides information about the actual performance parameters within the project supporting cellular network infrastructure on German highways. Thus, the paper shall give a deeper insight to the topic of HD Map supported highly automated driving to a broader audience. It especially shall provide future network planners with an idea of the requirements of highly automated vehicles to improve existing networks in the view of arising $5 \mathrm{G}$ network technologies.

The presented work is, therefore, structured as follows. In Section II a short overview about the existing Related work regarding HD Map supported highly automated driving is given. In Section III we present the general working concept for the data exchange between the vehicles and the data processing back end server, which collects the sensor data from the vehicles and provides map updates back to them. In Ko-HAF this data processing server is called Safety Server, as it enhances safety and comfort of the passengers while driving highly automated. In Chapter IV the testing area of Ko-HAF around the city of Frankfurt on the Main is described, where the cars are roaming and collecting data in real traffic situations. Section V illustrates the amount of data that is shared between each vehicle and the Ko-HAF Safety Server in both upload and download direction. Chapter VI and VII put those values in the context of the actual available bandwidth in both directions. Therefore, the vehicles measured the available network performance through their on-board communication unit as described in Section VI-A. Finally, in Section VIII we draw our conclusion from the obtained results of the project and propose future work.

\section{RELATED WORK}

\section{A. HD Maps}

Google's self-driving car project now better known as Waymo is one of the most well-known projects in the area of highly automated driving. For their driving experiments a precise HD Map has been created of the city of Mountain View in California where Google's headquarter is located. Due to the reliances of Waymos vehicles upon the map [4], [5] the cars are only able to drive in those areas which are covered by the map, not at any other given location. Similar approaches are conducted by the car manufacturers such as Ford [4] and Daimler [1]. Daimler, for example, conducted an automated test drive with one of their S-Class prototype vehicles along the historic track between Pforzheim and Mannheim, which Berta Benz, the company's founder's wife, has driven more than 125 years ago. The track is about $103 \mathrm{~km}$ long and consists of different road terrains such as inner city segments and country roads. To ensure the success of this test drive the S-Class relied on a digital map to support its own on board sensors. Map companies such as Here [7] and TomTom [6] address the enormous task to create HD Maps by using specialized measuring vehicles. However, those initially created maps have to be constantly updated due to changes in the road infrastructure such as construction sites and other frequent traffic events. Mapping companies and sensor manufacturers like Here and Mobileye [4] as well as research projects such as AutoMat [3] and our own Ko-HAF project therefore investigate the use of vehicles as sensor probes, which are able to collect sensor information to keep the maps up-to-date.

In the context of the newly emerging $5 \mathrm{G}$ cellular communication standard the highly automated driving also is seen as a major use case [2]. As the car's own sensor equipment produces huge amounts of raw data this might require fast communication and furthermore low latency to ensure the comfort while driving. However, most of the discussions in this area are currently based on hypothesis of future requirements. To the best of our knowledge this is one of the first publications to investigate the actual required amount of data for highly automated vehicles, to be supported via a back end enhanced HD Map, as the data is not transmitted in its raw format, but in an already processed and aggregated way.

\section{B. Connectivity maps}

In order to quantify the quality of the network a technique for measuring its throughput is required. In [8] Pögel and Wolf presented a concept called connectivity map to visualise and predict mobile network parameters for moving clients. To build such a connectivity map, mobile network parameters are collected and augmented with location information e. g. GPS coordinates. Then, all data points of an area are aggregated to so-called segments. Their first studies on $3 \mathrm{G}$ networks [8] took passive features like the Received Signal Strength Indication (RSSI) as well as active parameters like the bandwidth into account. As the parameters are fluctuating differently, an investigation on the length of map segments for the Channel Quality Indicator used in HSDPA networks was shown by Kelch et. at. in [9]. Another investigation on different segment building techniques was presented by Schmid et. al. in [10]. Schmid et. al. compared the accuracy of on-road segments with flexible and fixed lengths as well as a grid-based approach. Their results showed that the gridbased method have a similar accuracy compared to the fixedlength segment approach, but is much easier to be deployed. Additionally, there are actually two approaches for calculating and storing connectivity maps. The first is to set up a central database server like shown by Pögel and Wolf in [11], the second is to build personal maps on the client as introduced by Murtaza et. al. [12]. Although the second approach reuses the effort spent for communication, it has also disadvantages like the leak of data. In this paper, we focus on the server-based approach, as we see the connectivity map as an additional layer in the HD Map of the Ko-HAF Safety Server as shown in Section III. Furthermore the mandatory transmission of sensor 
data and map updates can be used to collect the connecitivity information free of charge as shown by Jormich el. at. [13].

\section{Ko-HAF SAFETy SERVER CONCEPT}

The aim of the Ko-HAF project is to investigate the next major step towards fully automated driving, which is highly automated driving at higher speeds. This degree of automation is characterized by the fact that the driver no longer has to permanently monitor the system. He can perform other tasks and is informed by the system, if a handover back to manual driving is required. To ensure the safety and the comfort of its passengers at high speeds, the vehicles need to be able to communicate via the cellular network to increase the area of observation beyond the range of their own on-board sensors. Traffic information such as traffic jams or black ice have to be exchanged between a map data providing back end server (so-called Safety Server) and the vehicle. Such information are required for anticipatory driving and route planning. If its current driving lane is affected by the received event, the vehicle is able to decide whether an alternative route should be taken or the driving can be made safer by reducing the vehicle's speed. The data exchange requires a suitable transmission protocol to send the data to the server and to receive updates of the car's high-definition map. In Ko-HAF, an extension of the SENSORIS protocol (version 2.0.2 [14]) has been developed to upload the sensor data from the vehicle to the server. This extension as well as other work in this area lead to a new SENSORIS version [15] that has the potential to be used in productive solutions in the area of vehicle sensor data provisioning.

An overview of the Ko-HAF communication flow is shown in Figure 1. A sensor data upload starts with a sensor detection event recorded by the vehicle system. This event is then sent to the Ko-HAF communication box (AP1 Box), where it can be queued to reduce the transmission overhead via the mobile network. In addition, the AP1 Box can also measure network parameters to monitor the connectivity of the vehicle. If the message queue is filled up or a high priority detection, like a traffic jam, is sent by the vehicle system, all data is transmitted to the server. There, it is stored in an input buffer for further processing. If a certain amount of collected sensor data is present in the input buffer, the aggregation can generate an updated part of the HD Map. This map is structured into different layers to distinguish between all kinds of dynamic and static parts of the map, such as construction sites or the connectivity layer. The layered map can also be updated by other data providers via an additional interface. To take the different requirements regarding latency into account, there are two ways of sending data to the vehicles. The first one is a state of the art request/response approach. It delivers map tiles or updates of them via a REST API to the vehicles. The second approach is used for dynamically occurring events such as accidents, which need to be sent as soon as possible to the vehicles. Therefore, a publish/subscribe protocol is used. The vehicles can subscribe themselves on their personal location area to get the dynamic traffic events, which are relevant for

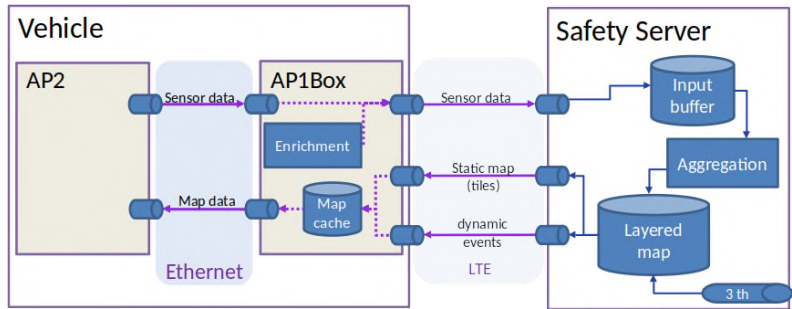

Fig. 1: Overview of the communication flow between vehicle and server in the Ko-HAF project.

them, directly when the server publishes them. That way, the HD Map inside the car can be used as an additional "virtual" sensor. To cover also areas without a good mobile network, a storage called map cache is implemented, which is able to store parts of the map needed that become relevant in the near future. To reduce the data transmission overhead, all exchanged data is decoded using Google Protocol Buffers ${ }^{3}$.

As a common hardware platform for the AP1 Box, an invehicle PC is used. It is equipped with a CAT 3 LTE module, which offers a peak upload rate of $50 \mathrm{Mbps}$ and a peak download rate of $100 \mathrm{Mbps}$. The module is using the MIMO mode with two antennas mounted on the roof of the vehicle to ensure the best possible signal reception quality. The data collection is done in a test area, which is presented in the following section.

\section{TEST AREA}

The Ko-HAF test area spans over a combined track length of about $275 \mathrm{~km}$ mostly consisting of highways and country roads including the driving area around the Frankfurt Rhein Main airport. The exact course of the tracks is indicated by Figure 3. It includes sections of the motorways A5, A661 and A3 to drive around the Frankfurt on Main city area as well as a larger section on the federal highway B45 in the eastern part of the map. These road sections have been selected as they resemble a quite busy traffic environment with a lot of commuting traffic in and around Frankfurt. Thus they provide challenging scenarios for the highly automated driving vehicles including quick changes of variable traffic signs and the clearance of the emergency lane for additional traffic in the daily rush hours. The test area is not only challenging in terms of the present traffic, but also in terms of the varying mobile network quality required for the data exchange as described in the following chapter.

\section{DATA CONSUMPTION}

As mentioned in Section III, the data consumption can be separated into two major categories: The upload shown in Section V-A and the download presented in Section V-B.

\section{A. Upload}

In general, the uploaded data contain sensor detections from the vehicle system. Sending all sensor readings directly to

\footnotetext{
${ }^{3}$ https://developers.google.com/protocol-buffers/
} 
the server would produce a huge amount of data and would require a lot of post processing computing power on the server. Hence, Ko-HAF developed a concept to pre-aggregate the raw sensor data on board the vehicle system. For example, instead of sending the detection of a single street sign multiple times to the server, a segment containing a road part and its surrounding area is uploaded. This allows the server to use the several objects contained in this message as correlating reference points to better locate the road part and its new features. Depending on the data provider, in the Ko-HAF project this message can contain street signs, boundary lines and lane markings as well as reflector posts. For the data amount of the message not only the features themselves play a role, but also their sample resolution. If e.g. a line is described with only a few points, in general the data required to transmit the feature is much less, but a single error in the measurement of one point has also a bigger impact on the total accuracy. As the feature accuracy resolution is an ongoing topic of research investigated in Ko-HAF, it was not possible to define the optimal amount of data points needed to build a line at the start of the project. Therefore, the used SENSORIS protocol does not define any maximum number of data points used to describe a lane marking. Figures $2 \mathrm{a}$ and $2 \mathrm{~b}$ show a visualisation of two distinct uploaded messages. Each black dot is a sample point of the detected line and contains the position offset to a reference point as well as a timestamp and a curvature value. Each measured point is represented by 90 bytes of data. Even though the data required for a single point seem to be very small, the transmitted messages quickly become large due to the quantity of sampled points. In Figure $2 \mathrm{a}$, the detected lines are sampled using a low sampling rate, while in Figure $2 b$ a higher sampling rate is used to describe the line. The pictures further show the trajectory of the vehicle indicated by green dots.

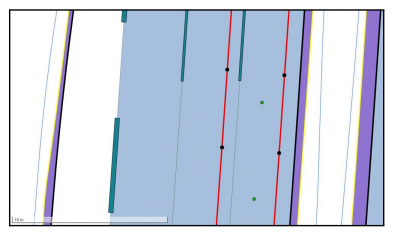

(a) A message containing line (b) A message containing line detection sample with a low detection sample with a high sampling rate sampling rate
Fig. 2: Visualization of an uploaded message containing line detection (red lines), the sampling points (black dots) and the vehicle trajectory (green dots).

Due to this very different rate of sampling points, the amount of sensor data bytes collected per $\mathrm{km}$ differs a lot between the data providers. For example, the data provider of Figure 2a produces between $170 \mathrm{kByte} / \mathrm{km}$ and $252 \mathrm{kByte} / \mathrm{km}$, while the provider of figure $2 \mathrm{~b}$ requires between $578 \mathrm{kByte} / \mathrm{km}$ and $1035 \mathrm{kByte} / \mathrm{km}$, four times as much as the average of the other provider. With ongoing sensor development and research on data aggregation, it is very unlikely that the vehicles of all car manufacturers will send exactly the same amount of data for the same road part in the future. Thus, to get a better understanding for the amount of data provided by the vehicles, an extrapolation using an average amount of $250 \mathrm{kByte} / \mathrm{km}$ as low data quantity and the peak amount of $1035 \mathrm{kByte} / \mathrm{km}$ as high data quantity, is shown in Section VII.

\section{B. Download}

Compared to the upload direction, the data consumption in the download direction is less. In the Ko-HAF data flow presented in Figure 1, there are two different channels used for the download data transfer. The first one is the static map channel. It is used regularly during driving, as the vehicle's HD Map static information like roads and traffic signs needs to be regularly fetched or updated. The map tiles used in the Ko-HAF system have a dimension of one square angular minute resembling approximately $2.20 \mathrm{~km}^{2}$ in the test area. The size of these tiles is between 12 kByte and 373 kByte. Assuming an average of $66 \mathrm{kByte}$ per tile results in a data rate of approximately $17.5 \mathrm{kBit} / \mathrm{s}$ in the case that a vehicle is driving in parallel to the meridian with a speed of 100 $\mathrm{km} / \mathrm{h}$. The second communication channel used in Ko-HAF is for dynamic and highly important events, like accidents or traffic jam messages. They do not occur repetitively and contain just a few descriptive bytes. Thus, we do not further take them into account for the data consumption calculation. Even though the download data consumption required in KoHAF is not very high, cooperative highly automated driving will enable the drivers to use other high data demanding services via the cellular network. This includes video or audio streaming as well as office use cases such as mailing or live video conferences. Thus, we consider such use cases in our evaluation as shown in Section VII. To put these requirements in relation to the actual achievable upload and download speeds in the cellular network, a detailed analysis of the achievable throughput in the Ko-HAF testing area is presented in the following.

\section{THROUGHPUT ANALYSIS}

The possible high peak LTE data rates, which the cellular providers use to advertise their networks, are most of the time not reached in a real deployment scenario as shown by Related Work [8], [11] and our own evaluation presented in the following. Actual real-world measurements performed by the vehicles are necessary to create a so-called "connectivity map" that showcases the actual achievable performance. In our personal opinion we see the connectivity map as an additional layer within the HD Map that is provided to the vehicles. With the help of this connectivity information the vehicles will be enabled to actively anticipate areas of good and bad network quality to schedule their mandatory data transmissions accordingly. Active measurements that are required to accurately create such a connectivity map layer could be conducted while transmitting the necessary sensor data in the upload direction and the map data updates in the down link direction. This concept is explained in detail in our 


\begin{tabular}{l|l}
\hline passive Data & $\begin{array}{l}\text { cellular network type, Receive Signal Strength Indica- } \\
\text { tor(RSSI), Signal to Noise Ratio (SINR), Reference } \\
\text { Signal Received Quality (RSRQ), Reference Signal } \\
\text { Received Power (RSRP), cell id, cell frequency }\end{array}$ \\
\hline active Data & $\begin{array}{l}\text { upload and download throughput in Mbit/s, round trip } \\
\text { time (RTT) }\end{array}$ \\
\hline additional Data & vehicle ID, vehicle position, vehicle speed, timestamp \\
\hline
\end{tabular}

TABLE I: Collected communication-related parameters from the Ko-HAF vehicle fleet.

earlier publication [13]. In the Ko-HAF project we wanted to further refine our obtained results regarding the question of the data requirements of highly automated vehicles with regards to such a connectivity map. Therefore, we investigated the actually achievable performance parameters in the Ko-HAF scenario around Frankfurt by using the vehicles as measuring probes of the network as described in the next section.

\section{A. Measurement setup}

One of the major contributions of the Ko-HAF research project is the common approach how cars are able to exchange sensor data and map updates with the back end Safety Server. The commonly developed concept as explained in Section III is further supported by a common communication hardware for all the cars in the Ko-HAF test fleet. This enables us, besides the data efficient transmission of sensor data uploads and map update downloads, to also collect detailed network quality parameters of the cellular network from all vehicles through their on-board communication units. That way we achieve a more detailed view on the currently available capacity of cellular networks, as a functional requirement of back end supported highly automated driving vehicles in future. The general concept to obtain the required measurements for the connectivity map layer is explained now.

While performing their test drives and exchanging data with the back end Safety Server, the Ko-HAF vehicles are constantly measuring their current cellular network quality. Therefore, the cars constantly collect their active and passive network quality parameters from the on-board LTE communication unit. This includes a multitude of different values, the most important ones are illustrated in Table II. Common passive parameters are the cell id of the cell tower, to whom the car is currently connected as well as the used transmission frequency band. Furthermore, the received signal strength is measured through different indicator values, such as the Receive Signal Strength Indicator (RSSI), the Signal to Noise Ratio (SINR), the Reference Signal Received Quality (RSRQ) and the Reference Signal Received Power (RSRP).

Besides those parameters the vehicle also performs cyclic active measurements to obtain the achievable up link and down link speed as well as the current latency of the cellular connection at its current position. The communication unit therefore sends 4 Megabytes of test data packets in the upload and download direction via TCP. To separate the transmissions from each other, a guard interval of 1 second is used in between. We decided to transmit 4 Megabytes of data to ensure that the TCP protocol is out of its slow start phase and able to reach the cellular networks peak bandwidth. The current round trip time of the connection is estimated through the arrival times of the TCP Syn and Ack messages of the 3-way handshake of TCP.

As all vehicles use the same communication hardware and antenna array, they collect comparable network quality parameters and therefore are able to contribute to a large data set that we investigate in the next section.

\section{B. Evaluation}

Figure 3 shows the mean average achieved down link bandwidth in the Ko-HAF testing area. Each color-coded segment resembles a circle of 500 meters on the track, for which the average out of all measurement values contained in it, is calculated. For our evaluation we have to take into account that the used communication equipment only achieves the LTE device category 3 (LTE cat. 3), which means it is able to achieve a maximum speed of $100 \mathrm{MBit} / \mathrm{s}$ in the down link and $50 \mathrm{MBit} / \mathrm{s}$ in the up link. The Ko-HAF box is not able to use higher modulation schemes and LTE advanced technology features like Carrier Aggregation, which have been introduced in newer device categories and would improve the overall achievable throughput rates. Therefore, we have to compare the achieved throughput measurements by taking the box capabilities into consideration. First of all it is clearly visible on the map that as comparable to other Related Work [8], [11] areas with good and bad network connection can quickly change. The connectivity layer in the HD Map enables the vehicles to actively react to these changes to enhance their overall transmission performance, compared to the common, but less-effective approach of reactive transmission adaptation. In our opinion active measurements are necessary to achieve accurate performance indicators in the map as the performance parameters like the down link and up link throughput as well as the latency of the connection are not only influenced by the passive network indicators like the received signal strength [16]. As cellular networks are a shared medium between all its users their peak performance also heavily depends on the actual active user base in the network's coverage area. This phenomenon is also visible in our data set when comparing the achieved down link bandwidth and the Reference Signal Received Power (RSRP) at the same spots as illustrated in Figure 4. The graphic shows the area around the Frankfurt airport in the south western area of the Ko-HAF test zone, as well as a section nearby the city of Obertshausen in the east of the map. In both cases the map indicates reasonably high RSRP values, but only low to medium achieved down link throughput values, which is possibly induced by a more active regular user base in those areas, compared to the remote areas of the highways.

To correlate our achieved measurement results with the vehicular traffic considered in Section VII, we extracted the relating area of the motorway A5 out of our collected data set. 


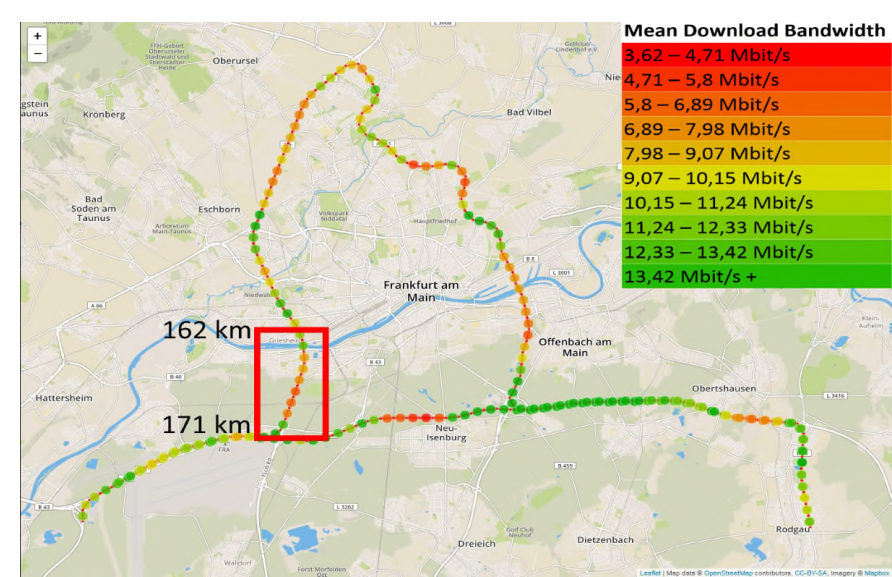

Fig. 3: Mean average achieved bandwidth in the down link direction. (O)penStreetMap contributors

This particular subset is marked by a red rectangle in Figure 3 . It is investigated in the following evaluation.

The 10 extracted segments within the rectangle range from street kilometre 162 in the north of the map to street kilometre 171 in the south. Figure 5 visualizes the achieved throughput values for up link and down link. First of all the huge variation in the achieved throughput values is visible. For the down link direction this varies from a maximum of around $40 \mathrm{Mbit} / \mathrm{s}$ down to about $1.75 \mathrm{Mbit} / \mathrm{s}$ at the same spot. For the up link data transmission LTE uses a different and more robust modulation scheme (Single Carrier Frequency Division Multiple Access - SC-FDMA) than for the down link (Orthogonal Frequency Division Multiplye Access - OFDMA). This is probably one of the reasons for the reduced variance in the achieved upload speed, which ranges from around $12.5 \mathrm{Mbit} / \mathrm{s}$ down to $1.75 \mathrm{Mbit} / \mathrm{s}$. Furthermore, it can be stated that the achieved average throughput values in this area are around 10 $\mathrm{Mbit} / \mathrm{s}$ for the down link and about $5 \mathrm{Mbit} / \mathrm{s}$ in the up link. In conclusion, it can be stated that the achieved throughput values vary largely and can decrease similarly to values well below $10 \mathrm{Mbit} / \mathrm{s}$. In the investigated area these achieved values also correlated with the achieved RSRP as indicated in Figure 7. High RSRP values at the beginning and the end of the scenario area also allowed higher achievable throughput values. The same tendencies are visible when comparing the RSRP and the achieved Round Trip Time (RTT) values indicated by Figure 6, although the changes in the Round Trip Time are not significant.

\section{THROUGHPUT NEEDS FOR AUTOMATED DRIVING}

To the best of our knowledge we are the first to investigate cellular network requirements for highly automated driving based on the real data requirements of a prototype fleet. Therefore we combine the obtained results of the data transmission schedule presented in Section $\mathrm{V}$ and the throughput measurements from Section VI into an analysis shown in the following. To evaluate a possible worst case scenario, we decided to select the area indicated by the red

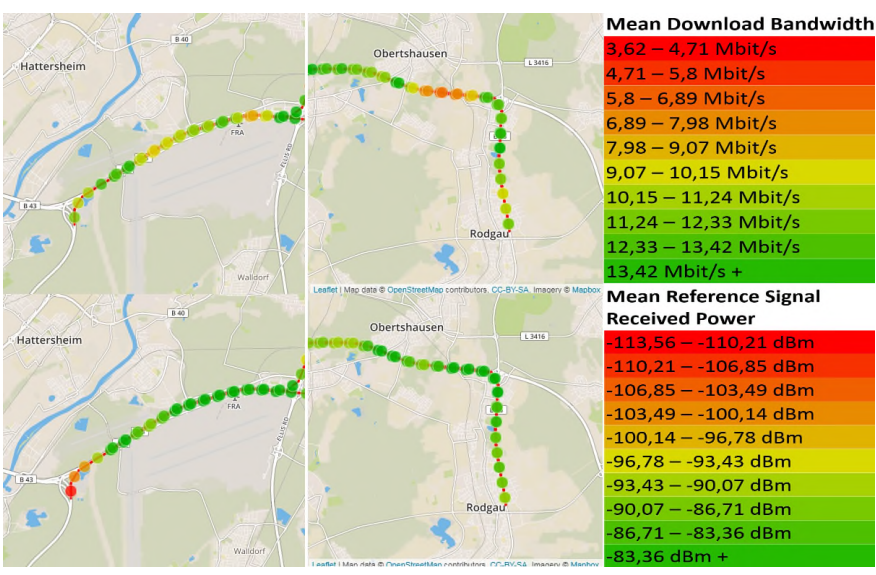

Fig. 4: Areas with no direct correlation between down link throughput and Reference Signal Received Power. COpenStreetMap contributors

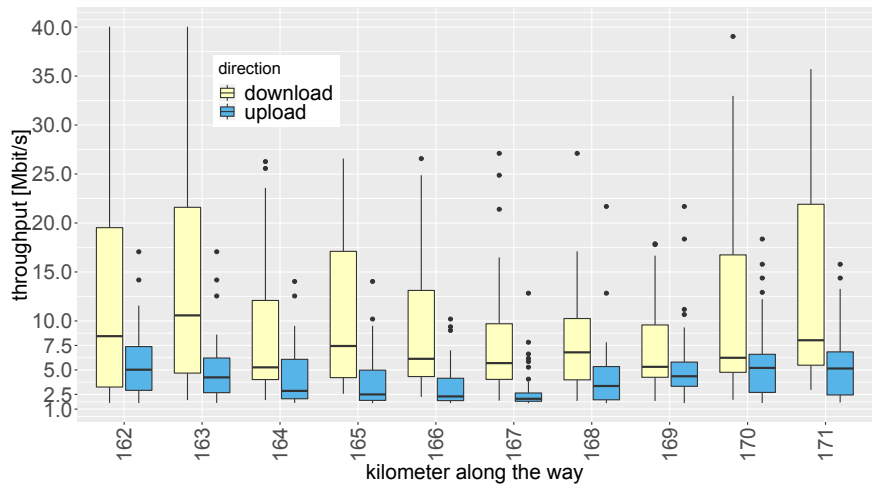

Fig. 5: Measured upload and download throughput in the investigated road section.

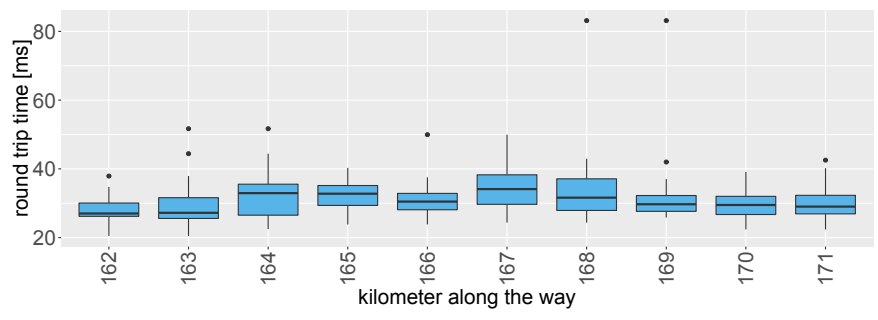

Fig. 6: Measured Round trip times in the investigated road section.

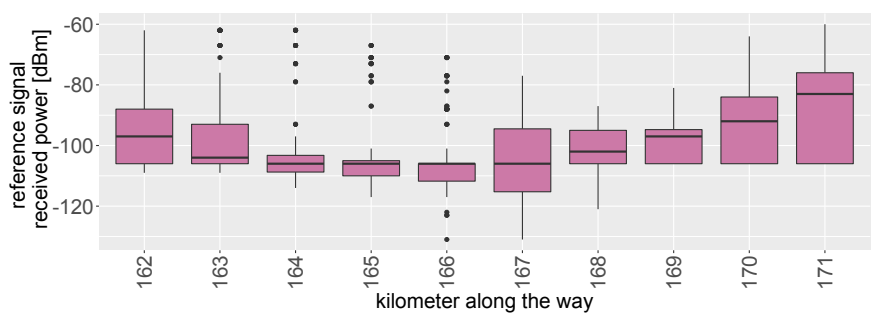

Fig. 7: Achieved Reference Signal Received Power (RSRP) in the investigated road section. 
rectangle of Figure 3 on the A5 near Frankfurt-Niederrad for our investigation. It represents an area with dense commuting traffic into and out of Frankfurt. According to the German Federal Road Research Institute (BASt) an average of more then 156,000 vehicles per day were counted at that place in 2016 [17]. For this specific area we investigate the cellular infrastructure requirements in the following.

The time required by a vehicle to pass the coverage area of a cell $c_{s}$, which can span several kilometers, can be calculated using the vehicle's speed $s$ (see Equation 1).

$$
c_{t}=\frac{c_{s}}{s}
$$

The market share of the provider $\alpha$ as well as the share of connections handled by the cell $\gamma$ also have to be taken into account, resulting in the amount of total used cells along the track. By using Equation 2 the required upload data rate for a single cell $c_{d}$ can be calculated. This equation can be simplified to a term only depending on the vehicles per cell $v_{c}$, the cell size $c_{s}$ and the data amount needed by one vehicle per kilometre $d$, resulting in Equation 4.

$$
\begin{array}{cc}
c_{d}= & \alpha \cdot \gamma \cdot v \cdot s \cdot c_{t} \cdot d \\
v_{c}= & \alpha \cdot \gamma \cdot v \\
c_{d}= & v_{c} \cdot c_{s} \cdot d
\end{array}
$$

Outgoing from this formula our evaluation of selected parameter combinations is shown in Figure 8. For the evaluation we investigated two different scenarios for the three German providers. Based on our measurements in the test area we achieved an average cell transmission range of $3.2 \mathrm{~km}$. At any position along the track two cells per provider were available, which leads to 6 cells in total, as the providers have a nearly equal market share [18]. Furthermore we evaluated a transmission range of $5.5 \mathrm{~km}$ as shown in the work of Afrić and Pilinsky [19] for a similar urban scenario, which uses only three cells in total. For our evaluation we assumed the uploaded data quantity to range between a value of 250 $\mathrm{kByte} / \mathrm{km}$ for a data provider sampling with a low rate and $1035 \mathrm{kByte} / \mathrm{km}$ for a provider sampling with a high rate as described in Section V-A. In Figure 8 the theoretical peak upload data rate for an LTE cell as well as our measured throughput value range as presented in Section VI is drawn as reference. The figure shows that for all scenarios that use the low data quantity the theoretical achievable peak throughput is sufficient enough to handle all data transmissions. Our actually measured throughput values however only achieve about $25 \%$ of this theoretical available throughput maximum. Assuming this available throughput the network upload capacity is too low for most of the scenarios. Only the transmission of a low data quantity could be realized in the investigated testing area, that hosted 6 different cells (blue dashed line). Either an increase in the amount of transmitted data (high data quantity) or a reduced available cell infrastructure ( 3 cells) as considered in the Related Work [19] would lead to an insufficient network capacity. Most critical are the peak traffic hours of the day in the morning and the evening ( 8 and 17 o'clock).

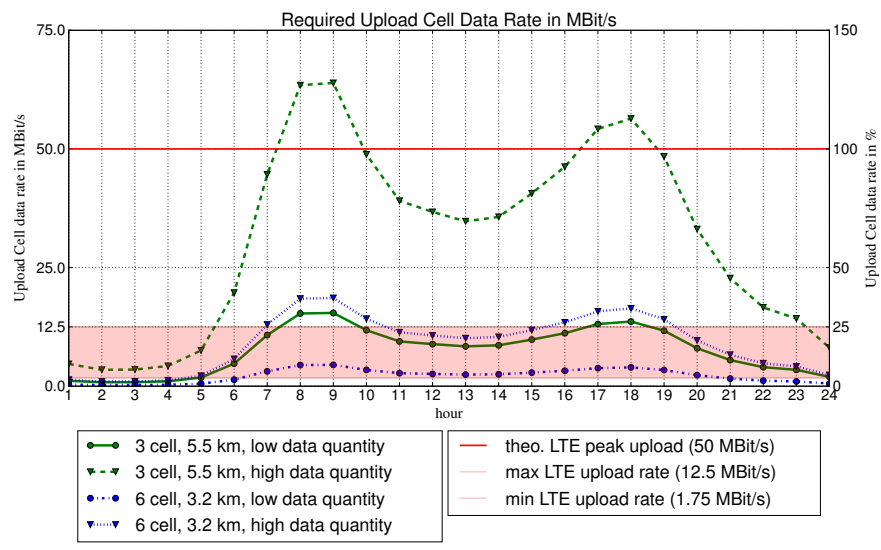

Fig. 8: Example extrapolation for the upload data rate based on counting spot data for A5 FRANKFURT-NIEDERRAD on April 11th, 2016. The different lines show combinations regarding the number of used cells, the cell size and the data consumption based on our measurements.

In a further evaluation step we investigated the download situation. Even though the map data transmission in the downlink for automated driving itself has been implemented very data efficient in Ko-HAF, with an average required bit rate of $17.5 \mathrm{kBit} / \mathrm{s}$ per vehicle, further services should be considered as well, when setting the passengers free from their driving task. The use of mobile services such as audio and video streaming will be made possible for the driver in future. Figure 9 , therefore, shows a selection of scenarios, which present the transmission of pure automated driving data as well as the transmission with additional audio $(320 \mathrm{kBit} / \mathrm{s})$ and video streams $\left(1,750 \mathrm{kBit} / \mathrm{s}\right.$ for $480 \mathrm{p}$ and $5,800 \mathrm{kBit} / \mathrm{s}$ for $\left.1080 \mathrm{p}^{4}\right)$. The figure shows that scenarios, where only the HD Map or the Map and an audio stream is downloaded, are possible with less than $10 \%$ of the theoretical maximum network capacity. In practice, considering our achieved throughput values this data transmission requires approximately $67 \%$ of the available network capacity. If the passengers use video streaming services, the achievable data rates are not sufficient enough anymore. In table II a more detailed view on the theoretical cellular capacity during the rush hour (15158 vehicles per hour) is shown. It presents that for HD video streaming even the theoretical maximum capacity of the present network infrastructure is not sufficient enough.

\section{CONCLUSION}

This work presents the obtained results of the German government-founded research project Ko-HAF regarding the question of communication requirements and data consumption for future back end supported highly automated vehicles. To the best of our knowledge this is one of the first works

\footnotetext{
${ }^{4}$ https://www.howtogeek.com/338983/how-much-data-does-netflix-use/
} 


\begin{tabular}{l|cc}
\hline $\begin{array}{l}\text { Frankfurt Scenarios } \\
(\mathbf{6} \text { cells, covering 3.2 } \\
\mathbf{~ k m})\end{array}$ & $\begin{array}{c}\text { Required Cell data } \\
\text { rate in MBits/s }\end{array}$ & $\begin{array}{c}\text { Required Cell data } \\
\text { rate in \% }(\text { of 300 } \\
\text { MBits/s) }\end{array}$ \\
\hline \hline ko-haf data & 1.41 & 0.47 \\
\hline ko-haf + audio data & 27.28 & 9.09 \\
\hline ko-haf + 480p data & 142.89 & 47.63 \\
\hline ko-haf + 1080p data & 470.30 & 156.77 \\
\hline ko-haf + 4k data & 1294.90 & 431.63 \\
\hline
\end{tabular}

TABLE II: Peak download data rate during the rush hour (15158 vehicles per hour).

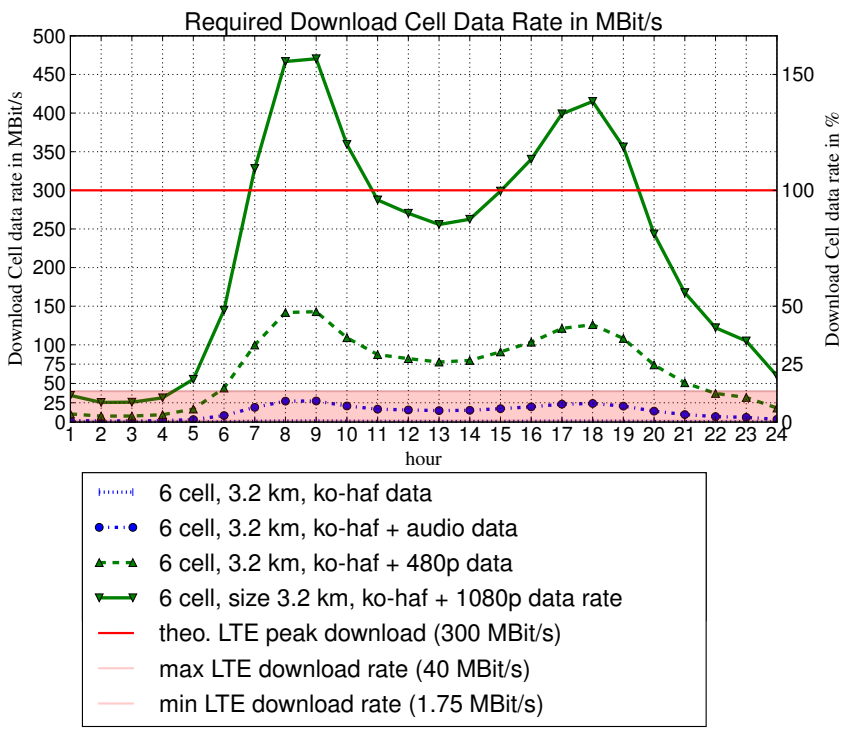

Fig. 9: Example extrapolation for the download data rate based on counting spot data for A5 FRANKFURT-NIEDERRAD on April 11th, 2016. The different lines show combinations regarding the number of used cells, the cell size and the data consumption.

to investigate the communication requirements of highly automated vehicles supported by actual productive and tested communication concepts to exchange map and sensor data between the vehicles and a data processing back end server. Therefore, we correlate a study regarding the data amount needed in the Ko-HAF project for the fleet supported HD Map generation, on which the cars rely while driving autonomously, with detailed quality analysis of the mobile network infrastructure in the Ko-HAF test area around Frankfurt, Germany. Our obtained results show that especially the data transmission speed required in the up link to exchange the sensor data of the cars can quickly exceed the capacity of the currently deployed cellular network infrastructure. Furthermore we investigate the down link requirements regarding the transmission of map updates and the possible further usage of other mobile services such as audio and video streaming that might exceed the currently available capacities when highly automated vehicles will become daily driving vehicles. With this work we hope to improve the awareness regarding the communication requirements of highly automated vehicles for the deployment of new network infrastructure in the scope of arising $5 \mathrm{G}$ technologies.

\section{ACKNOWLEDGMENTS}

This work results from the joint project Ko-HAF - Cooperative Highly Automated Driving and has been funded by the Federal Ministry for Economic Affairs and Energy based on a resolution of the German Bundestag.

\section{REFERENCES}

[1] J. Ziegler and et. al, "Making bertha drive - an autonomous journey on a historic route,' in IEEE Intelligent Transportation Systems Magazine, vol. 6, no. 2, 2014, pp. 8-20.

[2] H. G. Seif and X. Hu, "Autonomous driving in the iCity-HD maps as a key challenge of the automotive industry," in Engineering, vol. 2, no. 2, 2016, pp. 159-162.

[3] J. Pillmann and et al., "Novel common vehicle information model (CVIM) for future automotive vehicle big data marketplaces," in 2017 IEEE Intelligent Vehicles Symposium (IV), 2017, pp. 1910-1915.

[4] N. E. Boudette, "Building a road map for the self-driving car," 2017. [Online]. Available: https://www.nytimes.com/2017/03/02/automobiles/ wheels/self-driving-cars-gps-maps.html

[5] A. C. Madrigal. (2014) The trick that makes google's self-driving cars work - the atlantic. [Online]. Available: https://www.theatlantic.com/technology/archive/2014/05/ all-the-world-a-track-the-trick-that-makes-googles-self-driving-cars-work/ 370871/

[6] F. Holmes, "HD mapping 'takes ADAS to the next level', says TomTom," in Automotoive Mega Trends Magazin Q1, 2018.

[7] J. M. Gitlin. (2017) The most detailed maps of the world will be for cars, not humans. [Online]. Available: https://arstechnica.com/cars/2017/ 03/the-most-detailed-maps-of-the-world-will-be-for-cars-not-humans/

[8] T. Pögel and L. Wolf, "Analysis of operational 3g network characteristics for adaptive vehicular connectivity maps," in Wireless Communications and Networking Conference Workshops (WCNCW), 2012 IEEE. IEEE, 2012, pp. 355-359.

[9] L. Kelch and et al., "CQI Maps for Optimized Data Distribution," in Vehicular Technology Conference (VTC Fall), 2013 IEEE 78th. IEEE, 2013, pp. 1-5.

[10] J. Schmid, "Passive monitoring and geo-based prediction of mobile network vehicle-to- server communication," in Proceedings of the 14th International Wireless Communications and Mobile Computing Conference, 2018.

[11] T. Pogel and L. Wolf, "Optimization of GSM/UMTS inter-system handover times provided by connectivity maps," in Connected Vehicles and Expo (ICCVE), 2013 International Conference on. IEEE, 2013, pp. 641-646.

[12] G. Murtaza and et al., "Creating personal bandwidth maps using opportunistic throughput measurements," in 2014 IEEE International Conference on Communications (ICC). IEEE, June 2014.

[13] F. Jomrich and et al., "Iccomqs - intelligent measuring framework to ensure reliable communication for highly automated vehicles," in Vehicular Networking Conference (VNC), 2017 IEEE. IEEE, 2017, pp. 311-318.

[14] HERE. (2015) Vehicle sensor data cloud ingestion interface specification (v2.0.2). [Online]. Available: https://lts.cms.here.com/static-cloud-content/Company_Site/2015_ 06/Vehicle_Sensor_Data_Cloud_Ingestion_Interface_Specification.pdf

[15] ERTICO. (2018, July) Ertico coordinated platform releases first on vehicle-to-cloud data standard. [Online]. Available: http://erticonetwork.com/ ertico-coordinated-platform-releases-first-on-vehicle-to-cloud-data-standard/

[16] S. Sonntag and et al., "Mobile network measurements - it's not all about signal strength," in 2013 IEEE Wireless Communications and Networking Conference (WCNC), 2013, pp. 4624-4629.

[17] BAST. (2016) Automatische Zählstellen 2016, Dauerzählstelle: Frankfurt-Niederrad. [Online]. Available: https://www.bast.de/BASt_ 2017/DE/Verkehrstechnik/Fachthemen/v2-verkehrszaehlung/Daten/ 2016_1/Jawe2016.html?nn=1819490\&cms_detail $=6923 \& \mathrm{cms}$ map $=0$

[18] V. D. Consult. (2017) 19. tk-marktanalyse deutschland 2017. [Online]. Available: http://www.vatm.de/fileadmin/publikationen/PMs_Dateien/ 22_Marktstudie_2017/VATM_TK-Marktstudie_2017_181017.pdf

[19] W. Afrić and S. Z. Pilinsky, "Multipath fading and lte downlink cell size calculation," in Proceedings ELMAR-2013, Sept 2013, pp. 259-262. 